\title{
Significación de los marcadores infecciosos para identificar portadores de hepatitis B en donantes de sangre
}

\author{
Rosa Chiriboga-Ponce, ${ }^{1 *}$ Gabriela Cueva-Tirira, ${ }^{2}$ Carolina Crespo-Proaño, ${ }^{1}$ Andrés González-Rodríguez, ${ }^{1}$ \\ Pedro Pineda-Males ${ }^{3}$ y Mario J. Grijalva-Cobo ${ }^{1,4}$ \\ ${ }^{1}$ Centro de Investigación para la Salud en América Latina, Unidad de Referencia para Bancos de Sangre, Quito, Ecuador; ${ }^{2}$ Pontificia Universidad \\ Católica del Ecuador, Facultad de Ciencias Exactas y Naturales, Quito, Ecuador; ${ }^{3}$ Instituto Ecuatoriano de Seguridad Social, Hospital de \\ Especialidades "Carlos Andrade Marín", Quito, Ecuador; ${ }^{4}$ Infectious and Tropical Disease Institute, Department of Biomedical Sciences, Heritage \\ College of Osteopathic Medicine, Ohio University, Estados Unidos
}

\section{Resumen}

Introducción: La identificación de portadores del virus de la hepatitis $B$ en donantes de sangre es imperativo para evitar la transmisión de la enfermedad a través de transfusiones sanguíneas. Objetivo: Determinar si los donantes de sangre con resultados positivos de los marcadores serológicos $\mathrm{HbsAg}$ y anti-HBc eran portadores de ADN del virus de la hepatitis $B$. Métodos: Se recolectaron 12745 muestras de seis bancos de sangre ecuatorianos, las cuales fueron analizadas con pruebas serológicas para identificar los marcadores infecciosos HBsAg, anti-HBc, anti-HBs mediante prueba ELISA automatizada. Todas las muestras positivas para uno, dos o los tres marcadores fueron analizadas con técnica molecular para determinar la presencia de $A D N$ viral. Resultados: Se identificó que $27.5 \%$ de las muestras reactivas solo a anti-HBc y $100 \%$ de las muestras con resultados positivos de HBsAg/anti-HBc-lgM/lgG presentaron $A D N$ del virus de la hepatitis $B(p=0.001)$. Conclusiones: La elección de los marcadores de infección y los métodos de detección definen los resultados. Es importante la realización de dos pruebas serológicas y una molecular para identificar a los portadores del virus de la hepatitis $B$ y evitar su transmisión.

PALABRAS CLAVE: Hepatitis B. Anti-HBc. HbsAg. Portadores de virus de la hepatitis B. Donantes de sangre.

\section{Significance of hepatitis B surface antigen, IgM/lgG core antibody and hepatitis B virus DNA in blood donors}

\begin{abstract}
Introduction: Identification of hepatitis B virus carriers in blood donors is imperative in order to avoid transmission of the disease via blood transfusion. Objective: To determine if blood donors with positive results for serological markers $\mathrm{HBSAg}$ and anti-HBc were hepatitis B virus DNA carriers. Methods: 12,745 samples were collected from six Ecuadorian blood banks and analyzed for HBsAg, anti-HBC and anti-HBs infectious markers by automated ELISA. All samples that tested positive for one, two or all three markers were analyzed with molecular techniques to determine the presence of viral DNA. Results: $27.5 \%$ of the samples that were reactive for anti-HBc alone and $100 \%$ of those with positive results for $\mathrm{HbsAg}$ and $\mathrm{IgM} / \mathrm{IgG}$ anti-HBC were identified to contain hepatitis $B$ virus DNA $(p=0.001)$. Conclusions: The selection of infection markers, as well as the detection methods define the results. Performing two serological and one molecular test is important in order to identify hepatitis $B$ virus carriers and prevent its transmission.
\end{abstract}

KEY WORDS: Hepatitis B. Anti-HBc. HBsAg. Hepatitis B virus carriers. Blood donors.

Correspondencia:

*Rosa Chiriboga-Ponce

E-mail: rfchiriboga @ puce.edu.ec
Fecha de recepción: 24-01-2020

Fecha de aceptación: 23-07-2020

DOI: $10.24875 / G M M .20000032$
Gac Med Mex. 2021;157:37-42

Disponible en PubMed

www.gacetamedicademexico.com

0016-3813/@ 2020 Academia Nacional de Medicina de México, A.C. Publicado por Permanyer. Este es un artículo open access bajo la licencia CC BY-NC-ND (http://creativecommons.org/licenses/by-nc-nd/4.0/). 


\section{Introducción}

La seguridad de la calidad de los hemocomponentes utilizados en la medicina transfusional es de vital importancia en la práctica médica a nivel mundial; la transmisión del virus de la hepatitis B (VHB) aún constituye un problema de salud pública a pesar del uso de pruebas serológicas sensibles para la detección del antígeno de superficie y pruebas moleculares para la identificación de ADN viral. ${ }^{1}$ Debido a la necesidad de prevenir la transmisión de hepatitis $B$ a través de la sangre donada se introdujo el uso de nuevos marcadores serológicos como anti-core de VHB (IgM o $\operatorname{lgG}$ ) y pruebas moleculares; ${ }^{2}$ sin embargo, existe controversia en torno a la utilización de la prueba de anticore debido a reportes de un elevado número de resultados falsos positivos. ${ }^{2}$ También se ha señalado que la realización de la amplificación de ácidos nucleicos (NAT, nucleic acid testing) en pool resta sensibilidad a la detección del virus de la hepatitis B por la baja concentración de ADN del virus; es por ello que la prueba debe ser realizada por separado, lo que eleva los costos de su empleo en los servicios de sangre. $^{2}$

La prueba de anti-core (anti-HBc) es útil en la identificación de pacientes infectados o que han estado expuestos al $\mathrm{VHB}{ }^{3}$ adicionalmente, el anti$\mathrm{HBc}$ es considerado el único marcador detectable en donantes de sangre con infección resuelta, crónica o de cepas atípicas. ${ }^{1}$ La existencia de resultados positivos de anti-HBc y negativos del antígeno de superficie indica hepatitis oculta. Estados Unidos, Japón y Alemania tienen implementada la prueba para identificarlo, pero en Ecuador únicamente la realizan algunos bancos de sangre. El propósito de este estudio es determinar el significado de un resultado positivo de anti-HBc en ausencia del antígeno de superficie ( $\mathrm{HBsAg}$ ) en donantes de sangre ecuatorianos.

\section{Métodos}

Entre noviembre de 2008 y enero de 2010, fueron recolectadas 12745 muestras de suero de donantes de sangre de seis diferentes servicios de sangre ecuatorianos pertenecientes a las regiones costa y sierra; fueron analizados tres marcadores serológicos mediante técnica automatizada de ensayo por inmunoabsorción ligado a enzimas (ELISA, enzyme-linked immunosorbent assay). Todos los donantes de sangre firmaron el consentimiento informado (SENASFOS-10). Las muestras fueron codificadas sin nombres ni identificadores personales.

Para la detección serológica, todas las muestras de suero fueron analizadas con equipos comerciales automatizados para ELISA, siguiendo el protocolo de cada inserto. Los reactivos provinieron de tres casas comerciales diferentes:

- Inmunoensayos enzimáticos anti-HBc (total), de Genesis RMP 150/8 Génesis Abbott Murex, serie 6254.

- TECAN Schweiz AG Männedorf, analizador de inmunoensayo Axsym automatizado, serie 19540, montaje 37000.

- Abbott o Johnson \& Johnson. PIGEM0125/104.0.

Se realizaron pruebas adicionales para anti-HBs (EIA, Abbot Laboratories) en donantes con resultado reactivo de anti-HBc. Los criterios para la interpretación del resultado de las muestras se basaron en el punto de corte calculado de acuerdo con las instrucciones del fabricante de cada kit; todas las muestras que resultaron con un valor superior al del punto de corte se consideraron reactivas.

Todas las muestras que en las pruebas serológicas mostraron reactividad a cualquier marcador fueron analizadas mediante prueba molecular para identificar ADN-VHB; se empleó el equipo automatizado $\mathrm{COBAS}^{\circledR}$ Ampliscreen HBV (Roche Molecular System Inc., Branchburg, NJ, Estados Unidos). El ADN viral fue aislado directamente del plasma del donante mediante lisis de las partículas virales, para lo cual se utilizó el kit AmpliScreen HBV test N/P 21118323 123, reactivo de lisis MultiPrep (MPLYS); se usó el control interno (MPIC) compuesto por Tris- $\mathrm{HCl}<0-001 \%$ de ADN plasmídico no infeccioso y $0.1 \%$ de colorante de amaranto EDTA, el cual fue añadido a cada reacción para verificar la efectividad de la lisis, la extracción y la amplificación.

En cuanto a la amplificación por PCR, en el analizador COBAS $^{\circledR}$ AMPLICOR V0.2 la mezcla de reacción de amplificación (HBV MMX) estaba compuesta por tampón Tris, glicerol, $<0.001 \%$ de ADN polimerasa AmpliTaq $^{\circledR}$, sulfato de amonio, $<0.001 \%$ de AmpErase, $<1 \%$ de cloruro de magnesio, $<0.5 \%$ de Tween 20 y $0.09 \%$ de azida sódica. La mezcla de reacción tiene dos pares de cebadores $<0.05 \%$ de dATP, dCTP, dGTP, dUTP y < $0.001 \%$ de HBV-104UB, HBV-104D biotinlados), los cuales se amplifican de forma complementaria a las cadenas de ADN específicas de 104 pb de la región pre-core/core 
Tabla 1. Marcadores serológicos de hepatitis B de acuerdo con la región de procedencia, Ecuador 2008-2010

\begin{tabular}{|c|c|c|c|c|c|c|}
\hline \multirow[b]{3}{*}{ Región/servicio de sangre } & \multicolumn{6}{|c|}{ Marcadores serológicos } \\
\hline & \multicolumn{2}{|c|}{ HbsAg } & \multicolumn{2}{|c|}{ Anti-HBc IgM/lgG } & \multicolumn{2}{|c|}{ Anti-HBc IgM } \\
\hline & Reactivo/total & $\%$ (IC $95 \%)$ & Reactivo/total & $\%$ (IC $95 \%)$ & Reactivo/total & $\%$ (IC $95 \%)$ \\
\hline $\begin{array}{l}\text { Región costa } \\
\text { Cruz Roja Guayas } \\
\text { Cruz Roja El Oro } \\
\text { Maternidad E. Sotomayor }\end{array}$ & $\begin{array}{c}35 / 6100 \\
1 / 160 \\
3 / 222 \\
31 / 5718\end{array}$ & $\begin{array}{l}0.6(0.4-0.8) \\
0.6(-0.6-1.9) \\
1.4(-0.2-2.9) \\
0.5(0.3-0.7)\end{array}$ & $\begin{array}{c}26 / 382^{*} \\
10 / 160 \\
16 / 222 \\
N A\end{array}$ & $\begin{array}{c}6.8(4.3-9.3) \\
6.3(2.4-10.0) \\
7.2(3.8-10.6) \\
\text { NA }\end{array}$ & $\begin{array}{c}\text { NA } \\
\text { NA } \\
\text { NA } \\
15 / 5718\end{array}$ & $0.3(0.1-0.4)$ \\
\hline $\begin{array}{l}\text { Región sierra } \\
\text { Cruz Roja Quito } \\
\text { Hospital Carlos Andrade Marín } \\
\text { Hospital Metropolitano }\end{array}$ & $\begin{array}{l}18 / 6645 \\
8 / 4112 \\
2 / 973 \\
8 / 1560\end{array}$ & $\begin{array}{l}0.3(0.1-0.4) \\
0.2(0.1-0.3) \\
0.2(-0.1-0.5) \\
0.5(0.2-0.9)\end{array}$ & $\begin{array}{l}134 / 6645 \\
89 / 4112 \\
21 / 973 \\
24 / 1560\end{array}$ & $\begin{array}{l}2.0(1.7-2.4) \\
2.2(1.7-2.6) \\
2.2(1.2-3.1) \\
1.5(0.9-2.1)\end{array}$ & $\begin{array}{l}\text { NA } \\
\text { NA } \\
\text { NA } \\
\text { NA }\end{array}$ & \\
\hline Total & $53 / 12745$ & $0.4(0.3-0.5)$ & $160 / 7027$ & $2.3(1.9-2.6)$ & $15 / 5718$ & $0.3(0.1-0.4)$ \\
\hline
\end{tabular}

$\mathrm{HBsAg}=$ antígeno de superficie, $\mathrm{Anti}-\mathrm{HBC}=$ anticuerpos contra el core del virus de la hepatitis $\mathrm{B}, \mathrm{NA}=$ no analizado, IC $=$ intervalo de confianza

*En la estimación no se incluyeron dos muestras con resultados indeterminados.

conservada del genoma del VHB. La reacción de amplificación se realiza utilizando $<0.001 \%$ de polimerasa Taq (ADN polimerasa Thermus aquaticus), que es una enzima recombinante termoestable, para prevenir la presencia de amplicones. Se añadieron $50 \mu \mathrm{L}$ de la muestra a $50 \mu \mathrm{L}$ de mezcla de amplificación y se cargaron en el termociclador COBAS durante tres horas.

Las amplificaciones se llevaron a cabo con 30 ciclos a $90{ }^{\circ} \mathrm{C}$ durante 10 segundos y a $58{ }^{\circ} \mathrm{C}$ durante 25 segundos. Después de la etapa de amplificación, $25 \mu \mathrm{L}$ de solución desnaturalizante (DN4) que contiene hidróxido de sodio a $1.6 \%$, EDTA y azul de timol fueron agregados de forma automática a cada tubo de reacción para disociar el ADN amplificado; la mezcla fue colocada en las copas $D$ y se incubó en el termociclador de COBAS AMPLICOR durante 45 minutos a $91^{\circ} \mathrm{C}$. Después del último periodo de incubación, las copas $D$ fueron transferidas al fotómetro, donde se midió la absorbancia a $660 \mathrm{~nm}$. Se obtuvieron dos absorbancias de cada muestra: una del espécimen y la otra del control interno. El analizador COBAS AMPLICOR determina automáticamente el resultado del VHB de la siguiente manera: muestras con una absorbancia $<0.2$ y control interno $>0.2$ a $660 \mathrm{~nm}$ son consideradas negativas. Si la absorbancia para VHB es $\geq 0.2$, el resultado se considera positivo e indica la presencia de un segmento largo del virus B12 de 104 pb.

\section{Análisis de datos}

Los datos fueron analizados con el programa Stata, versión 11. Las prevalencias en las regiones costa y sierra se compararon utilizando chi cuadrada; un valor de $p<0.05$ se consideró estadísticamente significativo. Para la frecuencia de los marcadores virales de hepatitis B por región y bancos de sangre se utilizó estadística descriptiva con intervalos de confianza de $95 \%$ (IC). La evaluación del marcador anti-HBc se realizó comparando los marcadores serológicos HBsAg y anti-HBs con la PCR (detección de ADN viral) en muestras negativas y reactivas para la presencia del marcador anti-HBc; con la prueba exacta de Fisher se determinó su significación.

\section{Resultados}

Se recolectaron 12745 muestras de sangre, $52.1 \%$ en la región sierra y $47.9 \%$ en la región costa. Se identificó una prevalencia de $0.4 \%$ de muestras reactivas a HBsAg (IC $95 \%=0.3-0.5)$. Existieron diferencias significativas entre las dos regiones estudiadas: en la región costa se observó la mayor prevalencia que en la región sierra $(0.6 \%$ versus $0.3 \%$, respectivamente, prueba de $\chi^{2}, p=0.012$ ) (Tabla 1). La frecuencia de anti-HBc IgM/IgG fue de $2.3 \%$ (IC $95 \%$ = 1.9-2.6), mayor en la región costa, que en la región sierra $(6.8 \%$ versus $2.0 \%$, respectivamente, prueba de $\chi^{2}, p<0.001$ ); mientras que la prevalencia de anti$\mathrm{HBc} / \mathrm{lgM}$ fue de $0.3 \%$ (IC $95 \%=0.1-0.4$ ).

De las 7005 muestras negativas a HBsAg, 147 presentaron resultados reactivos a anti-HBc $\operatorname{lgM}$ e $\operatorname{lgG}$, que correspondieron a $2.1 \%$ (IC $95 \%=1.7-2.4$ ) (Tabla 2). La prevalencia general de HBsAg combinado con anti-HBc IgM/lgG fue de $0.2 \%$ (IC $95 \%=0.08-0.3$ ). Se observó reactividad aislada 
Tabla 2. Prevalencia combinada de HBsAg, anti-HBc IgM/lgG (total) y anti-HBc IgM entre donantes de sangre de las regiones sierra y costa

\begin{tabular}{|c|c|c|c|c|c|c|}
\hline \multirow[t]{2}{*}{ Marcadores virales } & \multicolumn{2}{|c|}{ Región sierra } & \multicolumn{2}{|c|}{ Región costa } & \multicolumn{2}{|c|}{ Total } \\
\hline & n/total & $\%$ (IC $95 \%)$ & n/total & $\%$ (IC $95 \%)$ & n/total & $\%$ (IC $95 \%)$ \\
\hline HBsAg negativo y anti-HBc negativo & $6503 / 6645$ & $97.9(97.5-98.2)$ & $354 / 380$ & $93.2(90.6-95.7)$ & $6857 / 7025$ & $97.6(97.2-98.0)$ \\
\hline HBsAg reactivo & $8 / 6645$ & $0.1(0.04-0.2)$ & $0 / 380$ & 0 & $8 / 7,025$ & $0.1(0.03-0.2)$ \\
\hline Anti-HBc-lgM/lgG (total) reactivo aislado & $124 / 6645$ & $1.9(1.5-2.2)$ & $23 / 380$ & $6(3.7-8.4)$ & $147 / 7025$ & $2.1(1.7-2.4)$ \\
\hline HBsAg reactivo y anti-HBc-lgM/lgG (total) & $10 / 6645$ & $0.2(0.05-0.2)$ & $3 / 380$ & $0.8(-0.1-1.7)$ & $13 / 7025$ & $0.2(0.08-0.3)$ \\
\hline HBsAg negativo y anti-HBc-IgM negativo & $N / A$ & $\mathrm{~N} / \mathrm{A}$ & $5674 / 5718$ & $99.2(99.0-99.5)$ & $5674 / 5718$ & $99.2(99.0-99.5)$ \\
\hline HBsAg reactivo & N/A & $\mathrm{N} / \mathrm{A}$ & $29 / 5718$ & $0.5(0.3-0.7)$ & $29 / 5718$ & $0.5(0.3-0.7)$ \\
\hline Anti-HBc-IgM reactivo & $\mathrm{N} / \mathrm{A}$ & $\mathrm{N} / \mathrm{A}$ & $13 / 5718$ & $0.2(0.1-0.4)$ & $13 / 5718$ & $0.2(0.1-0.4)$ \\
\hline HBsAg reactivo y anti-HBc-IgM & $N / A$ & $\mathrm{~N} / \mathrm{A}$ & 2/5718 & $0.03(-0.01-0.08)$ & 2/5718 & $0.03(-0.01-0.08)$ \\
\hline
\end{tabular}

Anti-HBC $=$ anticuerpos contra el core del virus de la hepatitis $\mathrm{B}, \mathrm{IC}=$ intervalo de confianza, $\mathrm{HBsAg}=$ antígeno de superficie. $\mathrm{N} / \mathrm{A}=$ no disponible.

a HBsAg en ocho muestras de la región sierra. El análisis estadístico mostró diferencia estadísticamente significativa (prueba exacta de Fisher, $p<0.001$ ) en la seropositividad de todos los marcadores de hepatitis B entre las dos regiones (Tabla 2). Trece de 5687 sujetos con resultados negativos de HBsAg fueron reactivos a anti-HBc IgM $(0.2 \%$, IC $95 \%=0.1-0.4)$.

Respecto a la detección molecular del VHB, en $100 \%$ de las muestras que presentaron reactividad a $\mathrm{HBsAg}$ y anti-HBc IgM/lgG se identificó ADN-VHB. La probabilidad de identificar ADN viral disminuyó a $33 \%$ en las muestras positivas de HBsAg y a $27.5 \%$ en las muestras positivas de anti-HBc IgM/lgG. En $100 \%$ de las muestras en las que se identificaron $\mathrm{HbsAg}$ y anti-HBc IgM se detectó ADN viral.

Se encontró ADN-VHB en $72.2 \%$ de las muestras con resultados reactivos a $\mathrm{HBsAg}$, mientras que la detección de ADN viral fue de $30 \%$ en las muestras con resultados positivos de anti-HBc IgM y negativos de HbsAg. Una muestra (0.1\%) en la que se identificó $A D N$ viral fue negativa a anti-HBc IgM/lgG y HBsAg. También se observó ADN viral en muestras reactivas a anti-HBs, marcador indicativo de inmunidad contra el antígeno de superficie del virus (Tabla 3).

\section{Discusión}

En 2015, la Organización Mundial de la Salud reportó que existían 275 millones de personas que viven con el virus de hepatitis $B$ y que se estimaban 887000 fallecimientos por esta infección, ${ }^{4,5}$ por lo cual constituye un problema de salud pública. La prevalencia en
Tabla 3. Detección de ADN viral y marcador de anti-HBs con combinaciones de pruebas de HBsAg y anti-HBc (total y $\operatorname{lgM}$ ) positivo/negativo

\begin{tabular}{|c|c|c|c|c|}
\hline \multirow{2}{*}{$\begin{array}{l}\text { Marcadores } \\
\text { virales }\end{array}$} & \multicolumn{2}{|c|}{ ADN viral } & \multicolumn{2}{|c|}{ Anti-HBs } \\
\hline & $\begin{array}{l}\text { Positivo/ } \\
\text { total }\end{array}$ & $\%$ & $\begin{array}{l}\text { Positivo/ } \\
\text { total }\end{array}$ & $\%$ \\
\hline $\begin{array}{l}\text { HBsAg y anti-HBc } \\
\text { lgM/lgG (total) } \\
\text { negativos }\end{array}$ & $1 / 699$ & 0.1 & $0 / 10$ & 0.0 \\
\hline HBsAg reactivo & $1 / 3$ & 33.3 & $0 / 3$ & 0.0 \\
\hline $\begin{array}{l}\text { Anti-HBc } \\
\text { lgM/lgG (total) } \\
\text { reactivo }\end{array}$ & $39 / 147$ & 27.5 & $94 / 147$ & 66.2 \\
\hline $\begin{array}{l}\text { HBsAg y anti-HBc } \\
\text { reactivos }\end{array}$ & $13 / 13$ & 100.0 & $2 / 13$ & 15.4 \\
\hline $\begin{array}{l}\text { HBsAg negativo } \\
\text { y anti-HBc-lgM } \\
\text { negativo }\end{array}$ & 0/509 & 0.0 & * & * \\
\hline HBsAg reactivo & $13 / 18$ & 72.2 & $0 / 18$ & 0.0 \\
\hline $\begin{array}{l}\text { Anti-HBc IgM } \\
\text { reactivo }\end{array}$ & $3 / 10$ & 30.0 & $3 / 10$ & 30.0 \\
\hline $\begin{array}{l}\text { Ambos } \\
\text { marcadores } \\
\text { reactivos }\end{array}$ & $2 / 2$ & 100.0 & $0 / 2$ & 0.0 \\
\hline
\end{tabular}

este estudio de donantes de sangre positivos a antígeno de superficie y anticuerpos contra el core del VHB (anti-HBc) fue de $0.2 \%$ y la de reactividad solo a anti-HBc fue de $2.1 \%$, más elevada que la encontrada en donantes brasileños en 2003 y 2016, que fue 
de $0.14 \%$ respecto a anti-HBc y $\mathrm{HBsAg}$, de $1.67 \%$ solo a anti-HBc ${ }^{6}$ y de 1.63 únicamente a $\mathrm{HbsAg}^{7}$ Reportes en otros países indican prevalencias sumamente altas: $3.5 \%$ de anti-HBc en Malawi, $8 \%$ en Camerún y de 4.6 a $17 \%$ de hepatitis oculta en el norte y sur de África, ${ }^{8}$ datos que demuestran la importancia de determinar el significado en los resultados obtenidos en los diferentes marcadores serológicos para establecer estrategias de tamizaje serológico.

Un resultado reactivo a anti-HBc aislado puede presentarse en varias condiciones clínicas, incluidas falsa reactividad, periodo de ventana, fase tardía de la infección o hepatitis oculta. ${ }^{3}$ En el presente estudio se detectaron 142 portadores únicamente de anti-HBc IgM/lgG y 10 de anti-HBc IgM, lo cual en estos últimos indicaba presunta infección aguda. En el estudio realizado por Zaccheaus et al. se estableció una prevalencia de $18.1 \%$ de donantes de sangre portadores solo de anti-HBc IgM, resultado relacionado con la probabilidad de que se tratara de un periodo de ventana seguido de una infección aguda o remota por VHB sin viremia persistente o persistencia viral, como sucede con la hepatitis oculta. ${ }^{9}$

El marcador serológico de anti-HBc IgM es el primero en detectarse en respuesta a la infección aguda por hepatitis $\mathrm{B}$ y puede ser el único en la etapa de ventana cuando se ha resuelto la antigenemia del virus y aún no son detectables los anticuerpos de protección (anti$\mathrm{HBs}) .^{9}$ Esta afirmación concuerda parcialmente con los resultados obtenidos en esta investigación de los 10 donantes de sangre que presentaron reactividad a anti$\mathrm{HBc}$ IgM: tres fueron reactivos a anti-HBs con presencia de ADN viral, lo cual es sinónimo de replicación, por lo tanto, la realización de la prueba serológica de anti$\mathrm{HBc}$ IgM en estos casos evitó la transmisión de la infección a través de sangre transfundida. A pesar de ello, Jeremiah et al. concluyeron que un resultado reactivo a solo anti-HBc no es sinónimo de infección por hepatitis, luego de la observación de que pacientes con resultados anti-HBc reactivo/HBsAg negativo y con exposiciones repetidas al virus no desarrollan la enfermedad. ${ }^{9}$ Sin embargo, se han reportado casos de transmisión de hepatitis $B$ tanto en transfusiones sanguíneas como en trasplante de órganos de donantes con resultados de $\mathrm{HBsAg}$ negativo/anti-HBc reactivo. ${ }^{10}$

Otros estudios afirman que la reactividad a anti-HBc es considerada como falsa positiva y se ha observado en poblaciones de baja prevalencia de hepatitis B, estimándose que puede presentarse de 10 a $50 \%$, relacionada con el uso de pruebas de mayor sensibilidad, ${ }^{11}$ dato que corrobora la existencia en este estudio de $72 \%$ de muestras que solo presentaron anti-HBc, pero que fueron negativas a $\mathrm{HBsAg} / \mathrm{ADN}$ viral, comportamiento serológico que puede deberse a una infección transitoria. Para asegurar esta afirmación se debería determinar si se trata de una hepatitis oculta a través de la cuantificación del anti-HBc como medio directo para distinguir las etapas de la infección. ${ }^{11}$

En las directrices europeas y americanas, la hepatitis oculta se define como la ausencia del antígeno de superficie y la presencia de ADN viral. ${ }^{11}$ En el presente estudio se encontró un donante $(0.1 \%)$ con ADN viral sin HBsAg ni anti-HBc y $27.5 \%$ de donantes con ADN viral y solo anti-HBc. Estos casos podrían tratarse de individuos portadores de hepatitis oculta, lo cual indica que para el tamizaje de donantes de sangre debería elegirse la combinación de pruebas de marcadores serológicos con prueba molecular, para facilitar la detección de la infección en los diferentes estadios y evitar la transmisión postransfusional del virus de la hepatitis $B$.

El uso de pruebas moleculares para la detección de ADN-VHB es controvertido especialmente en donantes de sangre, tanto por la carga viral baja o su presentación intermitente en casos de infección oculta. ${ }^{12}$ Estos hallazgos demuestran la complejidad de la identificación de hemocomponentes potencialmente infecciosos, especialmente en países con baja prevalencia, como Ecuador, mediante el empleo de un solo marcador serológico. En este estudio se observó que con la prueba de HBsAg se detectó únicamente a $33.3 \%$ de los donantes portadores de VHB y que $27.3 \%(n=39)$ no hubiesen sido detectados sin la prueba de anti-HBc.

La Organización Mundial de la Salud recomienda que cada país determine su estrategia para evitar la transmisión de hepatitis $\mathrm{B}$ a través de transfusiones. ${ }^{12}$ Para definir qué algoritmo debe ser utilizado se requiere investigar y analizar el significado de los resultados obtenidos. Con los hallazgos de este estudio y los de Sosa-Jurado et al. se concluye que es importante la determinación de anti-HBc como marcador serológico.

\section{Conclusiones}

Los resultados obtenidos al aplicar dos pruebas serológicas y la molecular facilitan la detección de donantes portadores del virus de hepatitis $B$ o que han tenido un contacto previo con el virus y así evitar la transmisión postransfusional. Para establecer la existencia de replicación viral en los donantes de sangre se debería 
incluir otros marcadores serológicos de infección que permitan el seguimiento y tratamiento.

\section{Agradecimientos}

Los autores agradecen la colaboración de los bancos de sangre de la red pública de salud y privada complementaria del Ecuador, por la participación y apoyo al desarrollo de este trabajo de investigación.

\section{Conflicto de intereses}

Ninguno

\section{Financiamiento}

Esta investigación fue financiada por la Secretaría de Educación Superior, Ciencia, Tecnología e Innovación y la Pontificia Universidad Católica del Ecuador.

\section{Responsabilidades éticas}

Protección de personas y animales. Los autores declaran que para esta investigación no se realizaron experimentos en seres humanos ni en animales.

Confidencialidad de los datos. Los autores declaran que siguieron los protocolos de su centro de trabajo sobre la publicación de datos de pacientes.
Derecho a la privacidad y consentimiento informado. Los autores declaran que en este artículo no aparecen datos de pacientes.

\section{Bibliografía}

1. Gessoni G, Beggio S, Barin P, Favarato M, Galli C, Valverde S, et al. Significance of anti-HBc only in blood donors: a serological and virological study after hepatitis B vaccination. Blood Transfus. 2014;12:s63-s68.

2. Karimi G, Zadsar M, Vafaei N, Sharifi Z, Falah Tafti. M Prevalence of antibody to hepatitis $B$ core antigen and hepatitis $B$ virus DNA in HBsAg negative healthy blood donors. Virol J. 2016;13:36.

3. Wu T, Kwok RM, Tran TT. Isolated anti-HBc: the relevance of hepatitis B core antibody-a review of new issues. Am J Gastroenterol. 2017; 112:1780-1788.

4. Sumer U, Sayan M. Molecular epidemiology of hepatitis B virus in Turkish Cypriot. Pol J Microbiol. 2019:68:449-456.

5. World Health Organization [Internet]. Suiza: Hepatitis B; 2021.

6. Salles NA, Sabino EC, Barreto CC, Barreto AM, Otani MM, Chamone DF. The discarding of blood units and the prevalence of infectious diseases in donors at the Pro-Blood Foundation/Blood Center of São Paulo, São Paulo, Brazil. Rev Panam Salud Publica. 2003;13:111-116.

7. Pessoni LL, Aquino ÉC, Alcântara KC. Prevalence and trends in transfusion-transmissible infections among blood donors in Brazil from 2010 to 2016. Hematol Transfus Cell Ther. 2019;41:310-315.

8. Cameroon-Fopa D, Candotti D, Tagny CT, Doux C, Mbanya D, Murphy EL, et al. Occult hepatitis B infection among blood donors from Yaoundé. Blood Transfus. 2019;17:403-408.

9. Jeremiah ZA, Idris H, Ajayi BB, Ezimah AC, Malah MB, Baba MM. Isolated anti-HBc-IgM antibody among blood donors in the semi-arid region of Nigeria. Hum Antibodies. 2011;20:77-82.

10. Ogunfemi MK, Olawumi HO, Olokoba AB, Kagu MB, Biliaminu SA, Durowade $K A$, et al. Prevalence of antibody to hepatitis $B$ core antigen among hepatitis $B$ surface antigen-negative blood donors in llorin, Nigeria: a cross-sectional study. Malawi Med J. 2017;29:32-36.

11. Moretto F, Catherine FX, Esteve C, Blot M, Piroth L. Isolated Anti-HBc: significance and management. J Clin Med. 2020;11;9:202.

12. Sosa-Jurado F, Rosas-Murrieta NH, Guzmán-Flores B, Pérez-Zempoaltecalt C, Sánchez-Torres AP, Ramírez-Rosete L, et al. Prevalence of serologic hepatitis $B$ markers in blood donors from Puebla, Mexico: the association of relatively high levels of anti-core antibodies with the detection of surface antigen and genomic DNA. Hepat Mon. 2016; 16:e36942. 